the circulation). Intra-muscular genetrans fer has been widely considered for this purpose, and the During paper now suggests that the oral route may also be useful.

In thelast few years, thefield of genetherapy has received somebad press. The initial and, in our view, unreasonable expectations have not been met, giving every opportunity for thelessenthusiastic to expresstheir reservations. Wehave previously stated our view that gene therapy is in fact progressing steadily and remains within the time frame of development of a commercial pharmaceutical product ${ }^{12}$. The During report lends credence to this view.

1. During, M.J. et al. Peroral gene therapy of lactose intolerance using an adeno-associated virus vector.
Nature Med. 4, 1131-1135 (1998).

2. Anderson, W.F. Human gene therapy. Nature $\mathbf{3 9 2}$, 25-30 (1998).

3. Hallek, M. \& Wendtner, C.M. Recombinant adenoassociated virus (rAAV) vectors for somatic gene therapy: Recent advances and potential clinical applications. Cytokines \& M olecular Therapy 2, 69-79 (1996).

4. Linden, R.M. et al. Site-specific integration by adeno-associated virus. PNAS 93, 11288-11294 (1996).

5. Balague, C. et al. Adeno-associated virus Rep 78 protein and terminal repeats enhance integration of DNA sequences into the cellular genome. J. Virol. 71, 3299-3306 (1997).

6. Conrad, C.K. et al. Safety of single-dose administration of an adeno-associated virus (AAV)-CFTR vector in the primate lung. Gene Ther. 3, 658-668 (1996).

7. Cheng, D.Y. et al. In vivo and in vitro gene transfer and expression in rat intestinal epithelial cells by E1-deleted adenoviral vector. Hum. Gene Ther. 8, 755-764 (1997).

8. Halbert, C.L. et al. Transduction by adeno-associ- ated virus vectors in the rabbit airway: Efficiency, persistence, and readministration. J. Virol. 71, 5932-5941 (1997).

9. Sahi, T. Genetics and epidemiology of adult-type hypolactasia. Scand. J. Gastroenterol. Suppl. 202, 7-20 (1994).

10. Corozza, G.R. et al. Beta-Galactosidase from Aspergillus niger in adult lactose malabsorption: A double-blind crossover study. Aliment. Pharmacol. \& Ther. 6, 61-66 (1992).

11. Lozier, J.N. et al. Gut epithelial cells as targets for gene therapy of haemophilia. Hum. Gene Ther. 8, 14811-14890 (1997).

12. Alton, E.W.F.W. \& Geddes, D.M. Gene therapy for cystic fibrosis: Steady progress, should do well. Eur. Respir. J. 10, 257-259 (1997).

Ion Transport Unit

National Heart and Lung Institute

Emmanuel Kaye Building

London SW 3 6LR, U.K.

e.mail: e.alton@ic.ac.uk

\title{
New clues to the emergence of flu pandemics
}

\section{The discovery of a new virulence mechanism in an influenza A (H1N1) virus may help to explain how flu viruses acquire virulence and why the 1918 flu pandemic was so devastating.}

A TTHE END of the First World War, which caused the deaths of about nine million people, there arrived an equally devastating event: the pandemic outbreak of Spanish flu, which killed more than 20 million peoplein the following two years. One wonders whether Wilson Smith, suffering from influenza about 15 years later in 1933, realized that the strain of influenza virus he carried-A/WS/33 (H1N1), a descendant of the Spanish flu virus-would yield potential clues to the extreme virulence of the 1918 pandemic strain. Using a laboratory-adapted version (WSN33) of the Smith strain, Goto and Kawaoka ${ }^{1}$ describe in a recent issue of the Proceedings of the National Academy of Sciences a new mechanism for cleaving viral hemagglutinin that confers increased virulence on the flu virus. The fascinating

\section{ERIC C.J. CLAAS ${ }^{1,2} \&$ Albert D.M.E. Osterhaus ${ }^{1}$}

question that arises from this work is whether this new mechanism is involved in the virulence of the Spanish flu virus, which caused the 1918 pandemic.

Hemagglutinin is the major surface glycoprotein of the influenza virus and enables it to attach to the host cell. Cleavage of hemagglutinin by host proteasesgenerating a hydrophobic tail that is essential for the fusion of viral and cell membranes ${ }^{2}$-is a prerequisite for virus replication and seems to be a key factor in the virulence of influenza viruses. Mammalian and avirulent avian flu virusesusually have a singlebasic amino-acid residue at their hemagglutinin cleavage site and depend on specific host proteases found in only one or two tissues for hemagglutinin cleavage ${ }^{3}$. In two avian influenza virus subtypes ( $\mathrm{H} 5$ and $\mathrm{H} 7$ ), the introduction of a stretch of basic amino acid residues near the cleavage site facilitated cleavage of hemagglutinin by subtilisinlike proteases found in all host tissues ${ }^{3}$. This resulted in highly virulent fowl plague viruses that invaded many tissues, causing a characteristic systemic pathology and high mortality.

Goto and Kawaoka describe a new way to facilitate cleavage of influenza virus hemagglutinin. They discovered that another major glycoprotein of the WSN 33 virus, neuraminidase N1, binds and sequesters the ubiquitous protease precursor plasminogen. This sequestration promotes production of high levels of plas-
The pig as a mixing vessel
Man as a mixing vessel?

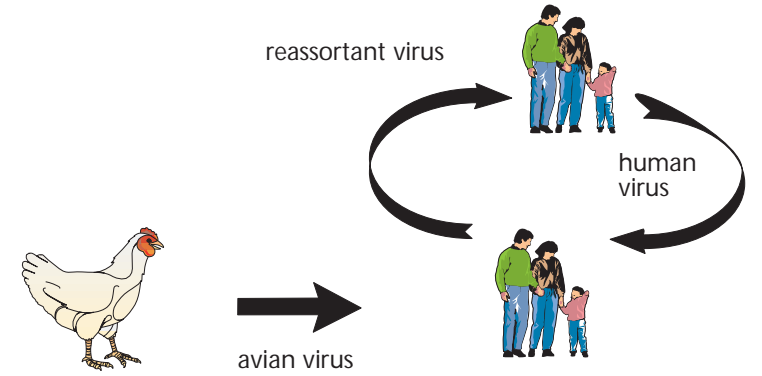

The pig may act as an intermediate host for the generation of human-avian reassortant influenza viruses with pandemic potential. Observations of humans infected with avian influenza A (H5N1) virus in Hong Kong in 1997 suggest that man himself may act as a 'mixing vessel'. 
min at the surface of the virus, which ensures highly efficient cleavage of hemagglutinin and enables the virus to invade a wide variety of tissues. They further demonstrate that plasminogen binding depends on the presence of a carboxyterminal lysine and the loss of an oligosaccharide side chain (due to a substitution of asparagine for arginine) at position 146 of the neuraminidase.

The 38 bases of neuraminidase sequence that have been determined for the 1918 virus strain ${ }^{4}$ exactly match those of WSN33, but unfortunately they do not fall within the plasminogen binding region. Plasminogen sequestration has not been found in the pandemic influenza A (H2N2) virus of 1957, the influenza A (H3N2) virus of 1968, or in any other influenza A (H1N1) virus, including the Smith strain. The multiple passages of WSN33 in chicken and mouse cells have selected for a virus with a neurovirulent phenotype $^{5}$ that in evolutionary terms is quite distant from its parent (Smith) strain and the 1918 virus. This raises the question of whether Goto and Kawaoka's finding is the result of the lengthy adaptation process in the laboratory or whether it could be acquired by (human) influenza viruses in nature. Sequence analysis of the appropriateregion of the Spanish flu virus neuraminidase may answer this question in the future. It is hoped that tissue samples from American soldiers who died of Spanish $\mathrm{flu}^{4}$ and from the recently exhumed bodies of 1918 flu victims in Brevig Mission (Alaska) and Spitsbergen (Norway) will help in this endeavor.

The Goto/Kawaoka results are of great interest because they indicate that acquisition of facilitated hemagglutinin cleavage may be a crucial feature in the identification of flu virus strains that are highly virulent. However, enhanced hemagglutinin cleavage is certainly not the only mechanism involved in acquisition of virulence. We and others have shown that an avian influenza A (H5N1) virus was responsible for the flu outbreak in Hong Kong last year that infected at least eighteen humans, six of whom died ${ }^{6,7}$. The H5 hemagglutinin of this particular virus contained a stretch of basic amino acid residues (also found in the virulent fowl plague viruses) that enabled efficient cleavage of hemagglutinin by the ubiquitous subtilisin-like proteases. The fact that this avian virus could replicate in humans was surprising, as previous studies had indicated in efficient replication of avian influenza viruses in experimentally infected humans ${ }^{8}$. Furthermore, in the individual Hong Kong cases, there was no evidence that the virus infected organs other than the respiratory tract $^{6}$, which would be possible if hemagglutinin had been cleaved by ubiquitous host proteases. The pandemic potential of theHong Kong virus was clearly hampered by its inability to be transmitted between humans ${ }^{7}$.

Extensive sequence analysis of all eight gene segments of different flu viruses revealed that the pandemic influenza strains of 1957 and 1968 are reassortants; that is, they are a combination of avian and human influenza viruses ${ }^{8}$. The gene segments of human origin obviously provide the virus with the ability to replicate in and spread among humans. The gene segments of avian origin, including that encoding hemagglutinin (which induces virus-neutralizing antibodies), produced a reassortant that would not encounter neutralizing antibodies in a human population unexposed to avian virus strains.

Studies on the host cell receptor specificities of avian, swine and human viruses ${ }^{9}$ as well as epidemiological data from swine influenza outbreaks ${ }^{10}$ support the notion that the pig is the likely intermediate for reassortment events between avian and human flu viruses ${ }^{11}$. The recent human influenza A (H5N1) cases in Hong Kong implicate man himself as an intermediate host for human-avian virus reassortment (see figure).

Predicting the time of the next influenza pandemic and the viral subtype involved is virtually impossible. Based on the observations in Hong Kong in 1997, one might expect a reassortant between the avian influenza $A$ (H5N1) virus and a circulating human influenza A (H3N2) or A (H1N1) strain. However, it may well be that only influenza viruses with an $\mathrm{H} 1, \mathrm{H} 2$ or H3 hemagglutinin can efficiently spread among humans. A repetitive cycle of human infection with a limited number of subtypes ( $\mathrm{H} 1-\mathrm{H} 2-\mathrm{H} 3)$ has been postulated $^{12}$. After antibodies against a certain hemagglutinin subtype have effectively disappeared from the population, a virus of that subtype could re-emerge. Bearing in mind that the 1977 re-emergence of influenza $A$ (H1N1) virus was not an actual antigenic shift ${ }^{13}$, a Eurasian-like swine HIN1 virus is a candidate for reintroduction as a recycled 1918 influenza A (H1N1) virus. Another possibility is the reappearance of influenza $A(H 2 N 2)$ virus. This subtype circulated over thirty years ago but only for a short period of time. Therefore, the prevalence of antibodies against this subtype in the population is limited.

It should be borne in mind that any influenza virus subtype capable of replicating in and spreading among humans could be a candidate for the next influenza pandemic. However, the only safe prediction we can make now is that another influenza pandemic will arrive. Therefore, continued surveillance of influenza in humans and animals, as well as the elucidation of mechanisms that determine the host range and virulence of flu viruses, will provide the best possible preparation for this inevitable event.

1. Goto, H. \& Kawaoka, Y. A novel mechanism for the acquisition of virulence by a human influenza virus. Proc. Natl. Acad. Sci. USA 95, 10224-10228 (1998).

2. Bullough, P.A., Hughson, F.M., Skehel, J.J. \& Wiley, D.C. Structure of influenza haemagglutinin at the $\mathrm{pH}$ of membrane fusion. $\mathrm{N}$ ature $\mathbf{3 7 1}, 37-41$ (1994).

3. Rott, R., Klenk, H.-D., Nagai, Y. \& Tashiro, M. Influenza viruses, cell enzymes and pathogenicity. Am. J. Respir. Crit. Care M ed. 152, S16-S19 (1995).

4. Taubenberger, J.K., Reid, A.H., Krafft, A.E., Bijwaard, K.E. \& Fanning, T.G. Initial genetic characterization of the 1918 "Spanish" influenza virus. Science 275, 1793-1196 (1997).

5. Francis Jr, T. \& Moore, A.E. A study of the neurotropic tendency in strains of the virus of epidemic influenza. J. Exp. Med. 71, 717-728 (1940).

6. Yuen, K. et al. Clinical features and rapid viral diagnosis of human disease associated with avian influenza A H5N1 virus. Lancet 351, 467-471 (1998).

7. Claas, E.C.J. et al. Human influenza A H5N1 virus related to a highly pathogenic avian influenza virus. Lancet 351, 472-477 (1998).

8. Webster, R.G., Bean, W.J., Gorman, O.T., Chambers, T.M. \& Kawaoka, Y. Evolution and ecology of influenza viruses. Microbiol. Rev. 56, 152-179 (1992).

9. Ito, T. et al. Molecular basis for the generation in pigs of influenza A viruses with pandemic potential. J. Virol. 72, 7367-7373 (1998).

10. Claas, E.C.J., Kawaoka, Y., De Jong, J.C., Masurel, N. \& Webster, R.G. Infection of children with avian-human reassortant influenza virus from pigs in Europe. Virology 204, 453-457 (1994).

11. Scholtissek, C., Burger, H., Kistner, O. \& Shortridge K.F. The nucleoprotein as a possible major factor in determining host specificity of influenza H3N2 viruses. Virology 147, 287-294 (1985).

12. Masurel, N.\& Marine, W.M. Recycling of Asian and Hong Kong influenza A virus hemagglutinins in man. Am. J. Epidemiol. 97, 44-49 (1973).

13. Nakajima, K., Desselberger, U. \& Palese, P. Recent human influenza A (H1N1) viruses are closely related to strains isolated in 1950. Nature 274, 334-339 (1978)

${ }^{1}$ Dept. of Virology and W HO National Influenza Centre

Erasmus University Rotterdam

P.O. Box 1738, 3000 DR Rotterdam

${ }^{2} D$ ept. of Virology,

CKVL, Leiden University M edical Center

P.O Box 9600

2300 RC Leiden

The Netherlands

email: Claas@virology.azl.nl 